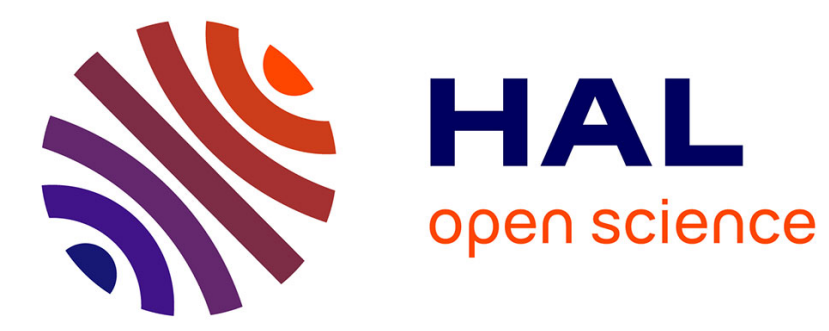

\title{
Exil, frontières et nation: José Miguel Carrera au Rio de la Plata
}

\author{
Edward Blumenthal
}

\section{To cite this version:}

Edward Blumenthal. Exil, frontières et nation: José Miguel Carrera au Rio de la Plata. Bulletin de l'Institut Pierre Renouvin, 2013, Les formes de l'exil dans le cadre d'émergence des États-nations, 38 (2), pp.17-31. hal-01540473

HAL Id: hal-01540473

https://hal-univ-paris3.archives-ouvertes.fr/hal-01540473

Submitted on 16 Jun 2017

HAL is a multi-disciplinary open access archive for the deposit and dissemination of scientific research documents, whether they are published or not. The documents may come from teaching and research institutions in France or abroad, or from public or private research centers.
L'archive ouverte pluridisciplinaire HAL, est destinée au dépôt et à la diffusion de documents scientifiques de niveau recherche, publiés ou non, émanant des établissements d'enseignement et de recherche français ou étrangers, des laboratoires publics ou privés. 


\title{
EXIL, FRONTIÈRES ET NATION : JOSÉ MIGUEL CARRERA AU RIO DE LA PLATA
}

\author{
Edward Blumenthal
}

IRICE | Bulletin de I'Institut Pierre Renouvin

$2013 / 2-N^{\circ} 38$
pages 17 à 31

ISSN 1276-8944

Article disponible en ligne à l'adresse:

http://www.cairn.info/revue-bulletin-de-l-institut-pierre-renouvin-2013-2-page-17.htm

Pour citer cet article :

Blumenthal Edward, « Exil, frontières et nation : José Miguel Carrera au Rio de la Plata », Bulletin de I'Institut Pierre Renouvin, 2013/2 №38, p. 17-31.

Distribution électronique Cairn.info pour IRICE.

(C) IRICE. Tous droits réservés pour tous pays.

La reproduction ou représentation de cet article, notamment par photocopie, n'est autorisée que dans les limites des conditions générales d'utilisation du site ou, le cas échéant, des conditions générales de la licence souscrite par votre établissement. Toute autre reproduction ou représentation, en tout ou partie, sous quelque forme et de quelque manière que ce soit, est interdite sauf accord préalable et écrit de l'éditeur, en dehors des cas prévus par la législation en vigueur en France. II est précisé que son stockage dans une base de données est également interdit. 
Exil, frontières et nation : José Miguel Carrera au Rio de la Plata

EDWARD BLUMENTHAL

L'indépendance des États d'Amérique du Sud, concomitant de la désintégration de l'Empire espagnol dans le cadre des guerres napoléoniennes, fut marquée par l'explosion, en leur sein, de forces centrifuges ${ }^{1}$. Suivant l'exemple ibérique, les villes capitales des anciens vice-royaumes - puis les villes provinciales - réclamèrent en effet leur souveraineté particulière, le pouvoir légitime symbolisé par le Roi d'Espagne étant vacant. Ce mouvement connu sous l'appellation de juntista - des juntes gouvernantes déclarées dans les villes - commença à Buenos Aires dès 1810 et, en 1811, au Chili. Dans ce territoire néanmoins, l'expérience ne dura que trois ans. La chute de la junte provoquée par les victoires des armées du vice-roi en 1814 sur les patriotes entraîna alors le départ de milliers de Chiliens qui traversèrent la frontière pour gagner Mendoza. Parmi eux se trouvaient l'ex-président de la junte José Miguel Carrera et sa famille.

Quelques années plus tard, entre 1817 et 1818, les forces du général José de San Martín, appuyées par le Directoire qui gouvernait Buenos Aires, toujours indépendant, conquirent le Chili. À la tête d'une partie des

1 Edward Blumenthal prépare actuellement une thèse ayant pour titre «Exil et constructions nationales, le cas de l'Argentine et le Chili au XIX siècle " au sein du laboratoire ICT (Identités Cultures Territoires) à l'Université Paris 7 Diderot, sous la direction de Pilar González Bernaldo. 
troupes, se trouvait un général chilien exilé en 1814, Bernardo O'Higgins, qui commandait des soldats eux-mêmes chiliens. Le général San Martín continua alors sa campagne au Pérou en 1819 pour lutter contre les Espagnols. Le Chili lui servit de base arrière et il nomma O'Higgins comme Directeur Suprême du Chili ${ }^{2}$. Cette chronologie coïncide avec l'émergence de revendications des villes du Rio de la Plata, désireuses de s'émanciper de la tutelle de Buenos Aires. C'est dans ce contexte que la trajectoire politique de Carrera au Rio de la Plata se déploie, avec l'objectif ultime de reprendre le pouvoir au Chili, d'abord des mains des Espagnols, puis de San Martín et O'Higgins.

Le fait d'exil ne résume pas la complexité de l'expérience de Carrera dans les Provinces Unies du Rio de la Plata ${ }^{3}$. Des réflexions pourraient également être menées sur les alliances qu'il tissa avec les gouverneurs des provinces du littoral et les Mapuches - des Indiens qui résidaient aux deux côtés de la Cordillère et qui avaient su maintenir leur indépendance des créoles tout au long de la colonie - ou encore sur sa relation conflictuelle avec le Directoire et San Martín. Néanmoins, il est pertinent de

2 Pour l'indépendance et le mouvement juntiste, voir Jeremy Adelman, Sovereignty and Revolution in the Iberian Atlantic, Princeton, Princeton University Press, 2006 ; François-Xavier Guerra, Modernidad e independencias: ensayos sobre las revoluciones hispánicas, México, Mapfre, 1993 ; Tulio Halperín Donghi, Revolución y guerra: formación de una elite dirigente en la Argentina criolla, Buenos Aires, Siglo Veintiuno Editores Argentina, 2005 ; Alfredo Jocelyn-Holt, La independencia de Chile : tradición, modernización y mito, Madrid, Mapfre, 1992 ; Jaime E. Rodríguez O., La Independencia de La América Española, México, Fondo de Cultura Económica, 2006 ; Geneviève Verdo, L'indépendance argentine entre cités et nation (18081821), Paris, Publications de la Sorbonne, 2006.

Pour l'exil, voir Yossi Shain, The Frontier of Loyalty: Political Exiles in the Age of the Nation-State, Ann Arbor, University of Michigan Press, 2005; Mario Sznajder et Luis Roniger, The Politics of Exile in Latin America, New York, Cambridge University Press, 2009. Mon argument doit beaucoup à ce dernier ouvrage qui postula que l'exil avait un rôle central dans les processus de construction de l'État national en Amérique latine. Pour la vie de J. M. Carrera, voir Bragoni Beatriz, José Miguel Carrera : un revolucionario chileno en el Río de la Plata, Buenos Aires, Edhasa, 2012 
réfléchir à la trajectoire politique de Carrera à partir de la problématique de l'exil. En effet, de la défaite des patriotes à Rancagua en 1814 jusqu'au moment de son exécution à Mendoza en 1821, il passa sa vie politique rocambolesque en dehors du Chili, tout en gardant comme objectif la reprise du pouvoir dans sa terre natale. En ceci, il faisait partie du mouvement plus grand des patriotes qui fuyaient les armées loyalistes en 1814. Dans son parcours individuel, on voit comment le combat à l'étranger liait Carrera au Chili, même dans l'absence.

À la tête d'un contingent militaire qui lui était attaché, Carrera essaya d'utiliser ses alliances avec les provinces argentines dans une lutte commune contre la domination de l'ex-capitale vice-royale Buenos Aires, sous la bannière d'un fédéralisme qui cherchait à affirmer les souverainetés provinciales. Mais Carrera élaborait aussi des alliances pour envahir le Chili, avec des acteurs de part et d'autre des Andes, comme les Indiens Mapuches et des soldats chiliens, patriotes et loyalistes, se retrouvant du " mauvais " côté de la frontière. Dans l'élaboration de ses alliances, le fait de la cordillère comme frontière n'était jamais loin, et le fait d'être Chilien gardait son importance.

La problématique de l'exil permet également de souligner l'imbrication des politiques argentine et chilienne après l'indépendance, et de déplacer le regard porté sur les conflits de 1820 et la désintégration des Provinces Unies, en ne les considérant pas comme un phénomène purement " argentin », mais impliquant aussi des Chiliens et des Mapuches des deux côtés des Andes. À partir de la trajectoire de Carrera au Rio de la Plata, on peut constater comment l'exil, outre sa simple dimension d'éloignement, connectait les nouvelles Républiques dans des rapports politiques imbriqués ${ }^{4}$.

4 Pour un exemple de l'imbrication de la politique dans les nouvelles Républiques dont une des manifestations fut la Confédération Pérou-Bolivienne, voir Natalia Sobrevilla Perea, The Caudillo of the Andes: Andrés De Santa Cruz, New York, Cambridge University Press, 2011. 
Alliances fédéralistes

En traversant les Andes après la défaite des patriotes chiliens en 1814, Carrera fut déchu de son commandement militaire par le gouverneur militaire du Cuyo, le général José de San Martín, et renvoyé à Buenos Aires avec ses frères. Une fois à Buenos Aires, il essaya de nouer des alliances avec les forces politiques porteñas qui lui permettraient de former une armée pour l'invasion du Chili, alors sous contrôle espagnol. Partageant sa haine pour San Martín avec Carlos María de Alvear, directeur des Provinces Unies en 1815, il améliora sa situation. Mais la chute d'Alvear l'obligea à partir pour les États-Unis, alors que ses frères se virent contraints d'aller à Montevideo, de l'autre côté du fleuve La Plata. Aux États-Unis, il arma une escadre pour effectuer la libération du Chili, mais en arrivant à Buenos Aires en 1817 il s'aperçut que San Martín et O'Higgins avaient déjà commencé l'invasion. Le nouveau directeur le fit emprisonner et saisit ses navires. Après sa libération la même année, il rejoignit ses frères en exil à Montevideo. Il y était entouré par des ennemis du gouvernement central et des Chiliens émigrés, tous décidés à renverser le Directoire. Son cercle incluait Alvear et ses amis et plusieurs Chiliens exilés $^{5}$. Leur double exil et leurs liens avec d'autres ennemis du Directoire, met en évidence l'imbrication des jeux d'alliance entre la Plata et le Chili, et souligne la stratégie argentine de Carrera: installer un pouvoir allié à Buenos Aires, à savoir Alvear, afin d'avoir l'appui du gouvernement pour ensuite envahir le Chili. Ses frères partirent en 1817 pour envahir le Chili, mais se firent exécuter avant d'avoir pu franchir la frontière à Mendoza, l'année suivante.

5 Tomás de Iriarte, Biografía del Brigadier General D. José Miguel Carrera, dos veces Primer Magistrado de la República de Chile, Buenos Aires, Imprenta de Mayo, 1863, p. 38-40. Iriarte, militaire de l'indépendance et exilé récidiviste, fut partisan d'Alvear. Les Chiliens incluaient Diego José Benavente et Manuel José Gandarillas, des partisans de Carrera bien avant l'émigration au Rio de la Plata. 
En 1820 l'autorité centrale du Directoire chuta lors de la bataille de Cepeda remportée par les forces fédéralistes des provinces de Santa Fe et de Entre Ríos, soutenues par Carrera. Par la suite, le traité de Pilar mit fin aux Provinces Unies, en établissant une alliance fédérale entre les provinces, les guerres ne se terminant pas pour autant. Comme nous le verrons par la suite, dans les guerres civiles qui opposèrent les fédéralistes aux forces de Buenos Aires, Carrera eut une place importante et une grande autonomie. Bien que dépendant des caudillos fédéralistes - des chefs militaires et dirigeants des provinces - pour les bases arrières, les munitions et les vêtements, Carrera était à la tête de ses propres troupes, composées essentiellement de Chiliens et d'Indiens.

Lorsqu'il est question de la trajectoire politique et militaire de Carrera au Rio de la Plata, il convient de rappeler que sa stratégie consistait à utiliser son influence auprès d'Alvear et des caudillos fédéraux en vue de réunir et de mobiliser autour de sa cause tous les soldats chiliens présents au Rio de la Plata. II semblerait que la plupart aient été soit des ex-prisonniers des armées espagnoles, soit des reliquats des soldats émigrés chiliens incorporés dans l'armée du Haut Pérou depuis $1815^{6}$. Selon Vicuña Mackenna, il y avait 2000 soldats chiliens au Rio de la Plata en 1820, dont 900 dans l'armée du Haut Pérou jusqu'au moment de sa dissolution en 1820. D'autres avaient servi à Buenos Aires et beaucoup de soldats en fuite après la bataille de Cepeda étaient des Chiliens ${ }^{7}$. Déjà en 1815 ,

6 L'armée de l'Haut Pérou (actuelle Bolivie) fut organisée par Buenos Aires pour expulser les forces espagnoles ancrées dans l'Haut Pérou, et exista de 1810 à 1820 , date à laquelle elle fut dissoute dans le contexte des guerres civiles.

7 Benjamín Vicuña Mackenna, El Ostracismo de los Carreras : Ios jenerales José Miguel i Juan José i El Coronel Luis Carrera. Episodio de La Independencia de Sud-America, Santiag, Impr. del Ferrocarril, 1857, p. 248 ; Tulio Halperín Donghi, Revolución y Guerra..., op. cit., p. 327 ; Barros Arana, Historia jeneral de Chile, Santiago, Rafael Jover, 1884-1902, vol. 9, p. 604. 
Carrera avait organisé une colonne de 500 soldats chiliens, dont l'armement était censé venir du Directeur Alvear ${ }^{8}$. En 1820, Carrera entama une stratégie de captation de ces soldats. II envoya un officier pour réclamer tous les soldats chiliens de l'armée du Haut Pérou, demande qui fut refusée par le colonel Juan Bautista Bustos, officier dans cette armée ${ }^{9}$. Après la chute du Directoire, Carrera essaya de mettre la main sur les Chiliens dans les milices de Buenos Aires ${ }^{10}$. En effet, le nouveau gouverneur de Buenos Aires, Manuel de Sarratea, lui permit de les rassembler dans la milice de la ville sous le commandement d'un Chilien ${ }^{11}$. De plus, pendant ses campagnes, on trouve même la présence de 15 ou 20 Chiliennes, femmes de soldats ${ }^{12}$. Dans les guerres contre Buenos Aires, à côté des forces de Santa Fé et Entre Ríos, on trouvait donc une armée chilienne, bien qu'indépendante et hostile à Santiago.

8 Benjamín Vicuña Mackenna, Ostraciscmo..., op. cit., p. 37.

9 José María Paz, Memorias Postumas del Brigadier General D. Jose M. Paz, Buenos Aires, Imp. de la revista, 1855, p. 38. Bustos et Paz étaient, tous les deux, à l'origine de la rébellion de Arequito. L'officier chilien, Urra, faisait partie du noyau d'officiers chiliens qui ont suivi Carrera jusqu'à la fin.

10 William Yates, un officier irlandais qui servait sous le commandement de Carrera, nota que ce dernier, lors de la bataille de Cepeda - la victoire des fédéralistes mit fin au Directoire -, empêcha la destruction d'une colonne composée de 600 Chiliens, pour pouvoir l'incorporer dans sa troupe. William Yates, "APPENDIX. A Brief Relation of Facts and Circumstances Connected with the Family of the Carreras in Chile, with some Account of the Last Expedition of Brigadier-General Don Jose Miguel Carrera, his Death, fyc. " in Lady Maria Callcott, Judas Tadeo de Reyes, et William Yates, Journal of a Residence in Chile, During the Year 1822, London, Longman, Hurst, Rees, Orme, Brown, and Green, 1824, p. 392.

11 Tomás de Iriarte, Biografía..., op. cit., p. 46; William Yates, «A brief relation... », op. cit., p. 399. L'officier chilien fut José María Benavente.

12 William Yates, "A brief relation... ", op. cit., p. 443. Pour les femmes dans les armées au Rio de la Plata, cf. Alejandro Rabinovich, "La Société Guerrière, Pratiques, discours et valeurs militaires au Rio de la Plata 1806-1852 ", thèse de doctorat sous la direction de Juan Carlos Garavaglia, EHESS, janvier 2010, p. 108 et sq. 
L'armée de Carrera : une force militaire chilienne?

Les partisans de Carrera maintenaient qu'il jouissait d'une réelle popularité parmi les Chiliens au Rio de la Plata. Selon Yates, les autorités de Buenos Aires donnèrent des passeports aux Chiliens pour l'ensemble des provinces, à l'exception de celle de Santa Fe, où justement se trouvait Carrera. Le fait que les Chiliens soient simplement passés par Montevideo pour se diriger vers Santa Fe ferait donc la démonstration de cette popularité. Un autre partisan de Carrera affirma qu'un appel de Carrera auprès des Chiliens de Buenos Aires généra 300 adhésions $^{13}$. Néanmoins, même si ces affirmations viennent de sympathisants de Carrera, peu objectifs sur cette question, les raisons de cette popularité restent ambiguës. D'une part, on pourrait argumenter que cette loyauté était plutôt personnelle et non nationale. C'est en effet la caractérisation la plus commune de Carrera et d'ailleurs une critique classique émise à l'encontre des caudillos en général ${ }^{14}$. D'autre part, les auteurs chiliens et les sympathisants de Carrera supposent implicitement que les Chiliens s'unirent à Carrera en raison de solidarités nationales. On peut donc suggérer, en nuançant, que le fait de constituer un corps unifié loin de leur patrie d'origine pouvait contribuer à leur identité en tant qu'unité militaire chilienne. En effet, l'hétérogénéité de la troupe - composée, comme nous l'avons vu, de loyalistes prisonniers et patriotes exilés - faisait des origines chiliennes un des seuls facteurs d'unité. Le récit de Yates démontre

13 Willliam Yates, «A brief relation... », op. cit., p. 414 ; Diego José Benavente, «D. José Miguel de Carrera », in Galería nacional, o, Colección de biografías i retratos de hombres celebres de Chile / escrita por los principales literatos del país, Santiago, Impr. Chilena, 1854, vol. 2, p. 35. Diego José Benavente, frère de l'officier du même nom, partagea l'exil à Montevideo avec Carrera et écrivit ce texte comme une défense de son nom.

14 Son contemporain le général Paz affirme qu'il « se trompa gauchement en pensant faire servir toute la République à ses buts personnels qui nous étaient complètement étrangers ", dans Memorias ..., op cit., p. 39. Le terme " personnel » est à prendre, dans le sens de Paz, par opposition à l'intérêt commun. 
également que même si Carrera obtînt et perdît des milices locales dans son passage par les provinces - indiquant que le facteur national n'était pas la seule attraction - le cœur de la troupe était composé de Chiliens émigrés ${ }^{15}$.

Si la combinaison de ces deux facteurs d'explication explique vraisemblablement les motivations des soldats, il est également important de noter que la terminologie de force militaire chilienne au Rio de la Plata n'implique pas le caractère étranger de cette force, en raison des imbrications politiques avec le Chili. L'alliance avec les fédéralistes passait certes par une communauté d'intérêts dans l'opposition à Buenos Aires et au Directoire, mais surtout conduisit Carrera à poser son opposition dans des termes fédéralistes. Dans des manifestes publiés en exil, Carrera insista sur le fait que le régime d'O'Higgins était essentiellement étranger. "Le Chili sera une Colonie de Buenos-Ayres comme il le fut de l'Espagne auparavant », argumente-t-i ${ }^{16}$. L'apparente contradiction ne devrait pas tromper. Certes, dans une certaine mesure, tout comme O'Higgins, il bénéficiait de soutiens de l'autre côté des Andes qui furent le produit des conflits au Rio de la Plata. Mais la différence réside justement dans leurs positions vis-à-vis de Buenos Aires, et dans les rancunes fédérales envers l'ex-capitale vice-royale qui essayait de légitimer sa domination actuelle en invoquant son statut passé. Carrera imagina le Chili comme «un des grands États de la Confédération du Sud" et non comme une "esclave [des] aristocrates de Buenos-Ayres ${ }^{17}$. II reprochait notamment à Buenos Aires d'avoir usurpé les ressources du Chili pour mener la guerre au Pérou, se référant ainsi au projet de San Martín et O'Higgins de poursuivre la lutte continentale contre les Espagnols. II est également possible que ce

15 Pour cette dynamique d'agrandissement des troupes par le recrutement des milices locales, voir Alejandro Rabinovich, "La Société Guerrière... », op. cit., p. 149.

16 José Miguel Carrera, Aviso a los pueblos de Chile, Montevideo, Impr. Federal, 1818, p. 1.

17 Ibid., p. 2. 
discours aidât aussi au maintien de la loyauté de ses troupes loyalistes d'origine chilienne.

C'est ici que l'on voit s'opérer le croisement entre la construction nationale et l'exil, catégories en construction qui se renforcent mutuellement. Carrera se présentait comme éloigné de sa patrie contre son gré. Sa motivation politique essentielle était donc le retour au pouvoir au Chili. En ce sens, il se comportait comme un exilé assez classique, mais dans une situation confuse et instable qui lui permit une plus grande autonomie que celle dont pourraient se prévaloir des émigrés 20 ou 30 ans après. Au même titre que les vagues d'exil ultérieures, du Chili ou du Rio de la Plata, il essayait en effet de monter une force capable d'envahir et de prendre le pouvoir. En revanche, la situation politique du Rio de la Plata diffère complètement. En l'absence d'un pouvoir étatique qui puisse l'aider, il est en effet obligé de l'organiser lui-même, avec les autres Chiliens. Or, les allégeances des acteurs sont variables, et même si le souhait premier de Carrera est de retourner au Chili, il continue à envisager d'autres configurations politiques possibles.

Cette complexité est soulignée par la rébellion de ses propres troupes loyalistes que Carrera dut affronter en 1821. Une division formée par des ex-prisonniers loyalistes espérait en effet traverser la montagne pour rejoindre la résistance loyaliste dans le sud du Chili ${ }^{18}$. Si le projet est identique - envahir le Chili - les idéaux sont eux différents. Cette rébellion souligne bien les ambiguïtés à la fois de la résistance loyaliste et du projet de Carrera, dont l'ennemi commun était pourtant identique, les forces de San Martín et d'O'Higgins. Deux conclusions peuvent dès lors être envisagées. D'une part, servir sous Carrera fut donc vraisemblablement perçu par les Chiliens comme un moyen de retourner chez eux. D'autre part, il est tout à fait possible que les loyautés "chiliennes " n'aient pas dépassé ce calcul, faisant abstraction de toute motivation politique.

18 William Yates, « A brief relation... », op. cit., p. 435. 
Acteurs et enjeux régionaux: Indiens et Chiliens, fédéralistes et loyalistes

En examinant la chute du Directoire en 1820, on retrouve une imbrication importante de la politique argentine et chilienne. San Martín est au centre de la politique chilienne et le soutien d'O'Higgins constitue le moteur de l'invasion du Pérou depuis le Chili en 1820 pour expulser les Espagnols de leur dernier lieu de pouvoir. Carrera était au centre des conflits des caudillos fédéraux, révolutionnant la situation du Rio de la Plata dans l'espoir de pouvoir envahir le Chili. Pour San Martín et Carrera, le but était identique même si les motivations divergeaient: échapper à la situation confuse au Rio de la Plata et aux conflits entre provinces. En effet, San Martín avait refusé l'ordre du directeur José Rondeau de retourner au Rio de la Plata en 1820 pour l'aider dans les guerres civiles contre les fédéralistes. C'est dans ce contexte qu'il faut comprendre le fédéralisme de Carrera, comme une reconnaissance empirique de l'importance du Chili au sein des conflits inter-provinciaux au Rio de la Plata. Des jeux d'alliances transfrontalières autant que des raisons idéologiques expliquent le fédéralisme de Carrera, mais il n'en reste pas moins un produit de ses sentiments anti-Buenos Aires. En effet, Carrera et ses soldats chiliens démontrent toute la complexité des enjeux régionaux - y compris de régions qui dépassent les frontières nationales actuelles - et comment les exilés pouvaient bouleverser les efforts des nouveaux États embryonnaires dont l'objectif était de détenir le monopole du contrôle de leurs frontières et leur population. Plus qu'un fédéraliste idéologique, Carrera chercha toute alliance transfrontalière qui pût l'aider dans son entreprise.

Ce jeu d'alliances se complexifie davantage quand on prend en compte les relations que Carrera eut avec les Indiens. Carrera n'était pas le seul caudillo à chercher une alliance avec eux, mais il était peut-être un des 
premiers $^{19}$. Selon Yates, des Indiens alliés à Buenos Aires approchèrent en premier Carrera au cours de 1820. Cette alliance était emblématique des imbroglios politiques frontaliers. Elle lui donnait en effet la possibilité de traverser la cordillère vers le Chili alors que, dans le même temps, il perdait ses alliances argentines et que les provinces lui interdisaient le passage de frontières. Les Indiens étaient en effet motivés par leur haine pour les porteños, et encouragés par un certain Guelmo, capitaine carrerista qui avait préféré s'exiler chez les Indiens plutôt que de servir sous O'Higgins ${ }^{20}$.

Nous nous trouvons donc en présence de trois groupes formant une alliance instable contre les unitaires au pouvoir à Buenos Aires: les Chiliens exilés qu'ils soient patriotes ou loyalistes, les fédéraux et les Indiens. Bechis a également identifié le contact principal de Carrera, à savoir Pablo Levenopán - dit «le Chilien » - un Indien arrivé dans la pampa avec d'autres caciques (chefs indiens) loyalistes vers 1818 et qui dévastait la frontière de la province de Buenos Aires. Le contact fut établi par le biais d'un loyaliste chilien, José Bielma, qui, avec son fils, avait demandé un passeport à Buenos Aires pour commercer avec les Indiens. Ceci démontre aussi que, contrairement à ce qu'affirme Yates, Carrera avait été en contact avec Levenopán depuis le début de 1820, quand il l'encourageait à attaquer la frontière pour diviser les forces de Buenos Aires $^{21}$.

Levenopán est un exemple classique de la migration des caciques « chiliens » vers les pampas après l'indépendance, encouragés par les

19 Martha Bechis, "Fuerzas indígenas en la política criolla Del Siglo XIX », in Caudillismos Rioplatenses: Nuevas Miradas a Un Viejo Problema, Buenos Aires, Eudeba, 2006, p. 299. Yates affirme néanmoins, que le gouverneur unitaire de Buenos Aires Rodríguez faisait de même, et implicitement que c'était déjà une pratique courante. William Yates, «A brief relation... », op. cit., p. 423424.

20 William Yates, « A brief relation... », op. cit., p. 416-417.

21 Martha Bechis, « Fuerzas indígenas... », op. cit., p. 299. 
loyalistes à saccager les villages à la frontière de Buenos Aires ${ }^{22}$. II est logique de penser que les loyalistes faisant partie des troupes de Carrera aidèrent à trouver de nouvelles alliances pour remplacer les fédéraux. Quoiqu'il en soit, les alliances avec les caudillos fédéraux étaient aussi un fardeau pour Carrera, et les Indiens représentaient une sorte de dernier refuge. La campagne de 1820 est pleine de tergiversations. À plusieurs reprises, alors qu'il s'apprête à traverser la frontière, Carrera doit y renoncer, étant appelé à l'aide par Alvear ou Ramírez ${ }^{23}$. Cette attitude, louée par ses sympathisants comme marque de fidélité de Carrera envers ses alliés, était emblématique, pour certains exilés, du danger de compromission avec la politique locale.

Si l'alliance avec Levenopán semble être aussi un moyen pour Carrera de s'approcher de la frontière, il faut noter que les traversées se font dans les deux sens. Selon Yates, la réputation de Carrera augmenta énormément pendant la campagne, et de plus en plus de caciques vinrent le rejoindre. "Deputies sent to Chile and the more distant nations requiring the attendance of caciques in the encampment of the Pichi Rey or little king (as such was the name they had given Carrera) naming a certain day for the junta of caciques to meet $»^{24}$. Mais il agissait davantage selon la

22 Pour le système mapuche transandin voir Martha Bechis, Susana Bandieri et Eduardo Cavieres, Cruzando la cordillera...: la frontera Argentino-chilena como espacio Social, Neuquén, Argentina : Centro de Estudios de Historia Regional CEHIR, Facultad de Humanidades, Universidad Nacional del Comahue, 2001.

23 Voir par exemple, Tomás de Iriarte, Biografía..., op. cit., p. 50-51 et p. 60 ; William Yates, "A brief relation... ", op. cit., p. 400-402.

24 «Députés envoyés au Chili et aux nations plus distantes demandant l'assistance des caciques dans le campement du Pichi Rey ou Petit Roi (tel était le nom qu'ils avaient donné à Carrera) désignant un certain jour pour la réunion de la junte de caciques ». William Yates, "A brief relation... », op. cit., p. 425426. Martha Bechis avance l'hypothèse qu'on l'appelait le Pichi Rey en opposition au Grand Roi, le roi d'Espagne, qui leur avait garanti leur autonomie jusqu'à l'indépendance, et que les loyalistes continuaient à promouvoir. En tant qu'étranger à la société mapuche, Carrera réussit à regrouper les caciques rivaux autour de lui, obtenant une centralisation inédite, quoique temporaire. 
logique des Indiens que selon la sienne ; au lieu de traverser vers le Chili, il était obligé de les accompagner dans leurs mallons, vers l'intérieur de la province de Buenos Aires, ce qui rendait la population hostile aux Chiliens $^{25}$. Les expériences transfrontalières étaient communes aux exilés et aux Indiens, ce qui peut être un facteur explicatif de leur alliance. Les Mapuches étaient à la croisée des routes commerciales reliant les deux versants des Andes que les loyalistes utilisaient pour attaquer les patriotes. Ainsi les Indiens furent des alliés qui contrôlaient les chemins rejoignant le Chili.

L'autre explication, plus classique, de son alliance avec les Mapuches, est liée au manque de solutions dont disposait Carrera. Pour l'historien libéral Vicuña Mackenna, écrivant en 1857 après son propre exil, cette alliance était symbolique du point de non retour dans lequel se trouvait Carrera, à savoir un exil permanent qui se terminerait avec sa mort prématurée. La campagne de Carrera avec les Mapuches devint ainsi une métaphore pour l'exil et le bannissement ${ }^{26}$. On constate la même situation sans issue chez les loyalistes ou les Mapuches, dont l'action était de plus en plus limitée par l'expansion des nouvelles républiques. Ainsi Carrera aurait-il affirmé à sa sœur qu'en cas d'échec de son retour au Chili : « Je serai Araucan et au moins les tyrans ne vivront pas tranquilles ${ }^{27}$. Dans la

Ainsi, les Mapuches ébauchèrent des structures politiques plus permanentes sous la pression du conflit entre Carrera, Buenos Aires et Santiago.

25 Tomás de Iriarte, Biografía..., op. cit., p. 56 ; Iriarte l'excuse en disant qu'avec 150 soldats "il ne pouvait pas contenir des Indiens furieux ». Yates fait de même en rajoutant que le gouverneur Rodríguez avait utilisé les Indiens avec des propos similaires, " A brief relation... », op. cit., p. 423-424.

26 El Ostracismo..., op. cit, p. 253 et p. 258-259 ; José Luis Rénique, « Benjamín Vicuña Mackenna : Exilio, Historia y Nación », in Carmen Mc Evoy Carreras et Ana María Stuven, La República Peregrina: hombres de armas y letras en América del Sur, 1800-1884, Lima, Instituto de Estudios Peruanos, Instituto Francés de Estudios Andinos, 2007, p. 499. Notamment, Vicuña Mackenna fut exilé avec le fils de Carrera, à la suite de leur participation à la révolution manquée en 1852.

27 Cité dans Martha Bechis, « Fuerzas indígenas... », op. cit., p. 300. 
trajectoire politique de Carrera, la traversée des frontières - nationales, provinciales ou entre Indiens et créoles - joua donc un rôle non négligeable dans les processus menant à la construction de ces mêmes frontières. En effet, un caudillo chilien « étranger » avec sa propre armée était un des acteurs principaux de la dissolution des Provinces Unies et l'établissement de souverainetés provinciales. Pour la première fois, celuici regroupa autour de lui des groupes indiens autonomes dans une construction politique plus large que celui organisé autour d'un $\operatorname{chef}^{28}$. Sa dernière campagne est de ce point de vue emblématique de la formation de nouvelles identités politiques, nationales et indiennes, de part et d'autre des Andes.

Ceci se vérifie aussi à travers la réaction des gouvernants chiliens. Carrera était en communication avec ses partisans au Chili, qui l'encourageaient à traverser la frontière au plus vite pour appuyer leur révolution. Cependant la saison n'était pas adéquate et les demandes de ses alliés l'en empêchaient. Dans le même temps, les caudillos fédéralistes demandaient tous son aide ${ }^{29}$. De plus, O'Higgins envoyait de l'argent aux gouverneurs de Córdoba, Mendoza, San Luis et San Juan, non seulement pour lever des forces afin d'éliminer Carrera mais aussi au profit d'une force chilienne au Cuyo. Selon Yates, cette dernière fut cependant rappelée à la frontière parce que O'Higgins doutait de la loyauté de ses soldats. Toujours selon le soldat irlandais, le gouverneur de Mendoza reçut la « légion du mérite » du Chili et la paie d'un général de brigadier du Chili

28 Comme on l'a vu, c'est l'interprétation de Bechis de l'alliance entre Carrera et les Mapuches, et qui se verra répéter quelques années après autour des Pincheira, des frères loyalistes qui continuèrent à se battre contre les créoles argentins ou chiliens - en alliance avec les Mapuches jusqu'aux années 1830. Pour un autre exemple d'un essai de construction d'un projet politique mapuche plus grand sur les mêmes réseaux pour affronter les défis de l'état-nation voir Julio Vezub, Valentín Saygüeque y la "gobernación indígena de las Manzanas ». Poder y etnicidad en la Patagonia septentrional (1860-1881), Buenos Aires, Prometeo Libros, 2009.

29 William Yates, "A brief relation... », op. cit., p. 401. Selon Yates, O'Higgins bannit plus de 40 partisans de Carrera. 
pour avoir ordonné l'exécution de Carrera $^{30}$. Le gouvernement chilien tentait en effet de sécuriser la frontière comme il le pouvait et de manière à limiter les menaces extérieures. II fut, en outre, obligé d'avoir recours aux gouvernements provinciaux, de l'autre côté de la cordillère. Néanmoins, les passages d'un côté à l'autre de la frontière sont constants: passages d'argent, groupes de Mapuches, nouvelles de révolutions manquées; seule la colonne de Carrera n'arriva pas à traverser.

\section{Exil et frontières}

Avec l'échec du projet politique de Carrera, on entrevoit un premier pas vers la consolidation d'une frontière entre le Chili et l'Argentine. C'est la collaboration entre le gouvernement chilien et les gouvernements provinciaux argentins qui conduisit à la chute de Carrera. En effet, la frontière politique entre le Chili et les provinces argentines s'avéra plus concrète dans la mesure où les autorités des deux côtés des Andes empêchèrent Carrera de la traverser. Le fait d'être un corps unifié se battant en terre étrangère aurait vraisemblablement donné un caractère national aux troupes de Carrera d'origine transandine, malgré la diversité de ces forces - patriotes, loyalistes, Mapuches - et même si le doute subsiste dans le cas des Indiens. Néanmoins, les imbrications entre les politiques argentine et chilienne soulignent la faiblesse de cette frontière, et le fait que des outsiders comme Carrera, les Mapuches ou encore les monarchistes pouvaient toujours la traverser, concevant des projets politiques alternatifs. Cette étrange coalition de patriotes, de fédéralistes, de loyalistes et d'Indiens traversait d'une manière ou d'une autre les nouvelles frontières, pratique que les nouveaux États nationaux ont par la suite bien du mal à éradiquer.

$30 \quad$ Ibid., p. 438-471. 